\section{Color in Dentistry A Clinical Guide to Predictable Esthetics}

Authors: Stephen J. Chu / Rade D. Paravina / Irena Sailer / Adam J. Mieleszko Publisher: Quintessence Publishing, Germany

Language: English

ISBN: 978-0-86715-745-1

Edition: $1 / \mathrm{e}$

Publish Year: 2017

Pages: 256, illustrated

Price: $108.00 €$

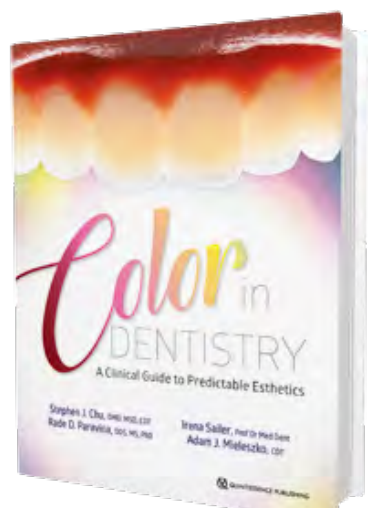

\section{Florin-Eugen}

Constantinescu

DMD, PhD Student

Holistic Dental \& Medical Institute of

Bucharest-ROPOSTURO, Bucharest,

Romania

e-mail:

drfflorin.constantinescu@gmail.com

Choosing the color in dentistry is a challenge for every practitioner. In time, a number of authors have dealt with this issue.

The book entitled "Color in Dentistry. A Clinical Guide to Predictable Esthetics" brings new insights into the subject, the materials used and the new technologies to which practitioners have access. It is an update on color education and training. The book is divided into twelve chapters. After the presentation of color education and training, the physics of color, color perception and color mixing are developed to facilitate their understanding. Chapter three presents data on the elements that affect the choice of the optimal color: illumination, clinical lighting challenges, contrast effects and optical illusions, viewer-associated effects, and other appearance attributes. The importance of evaluating the optical properties of teeth (white), gum (pink) and skin are developed in the chapter called The United Colors of Dentistry. The fifth chapter discusses conventional visual matching, shade guides, shade matching factors, and shade matching protocol. Chapter six talks about the development of technology-based shade systems, color measurement, workflow and protocol. The following chapters introduce camera system equipment, principle-based concepts, image management and color communication to better understand digital photography. The material selection for the final esthetic outcome in dentistry is developed in Chapter 8 . The following chapter presents color-matching strategies for discolored non-vital and vital tooth stumps as well and gingival, implant abutment and periimplant soft tissue. The following chapters describe in depth the esthetics with pink restorative materials and steps to a successful shade match. The last chapter sums up the presentation of 24 clinical cases from the simplest, such as shade monitoring for tooth whitening cases to the most complex as implant-retained silicone auricular prostheses. The book contains as well an appendix, an index, is richly illustrated with 890 images and helps facilitate communication between dentists and technicians.

By reading "Color in Dentistry" we understand the best protocol to follow in choosing the color for any clinical situation we want to restore as best we can.

DOI: 10.25241/stomaeduj.2018.5(2).bookreview.1 